\title{
How Does Ulnar Shortening Osteotomy Influence Morphologic Changes in the Triangular Fibrocartilage Complex?
}

\author{
Yoshiaki Yamanaka MD, Toshiyasu Nakamura MD, PhD, \\ Kazuki Sato MD, PhD, Yoshiaki Toyama MD, PhD
}

Received: 4 February 2014 / Accepted: 25 July 2014/Published online: 13 August 2014

(C) The Association of Bone and Joint Surgeons \& 2014

\begin{abstract}
Background Ulnar shortening osteotomy often is indicated for treatment of injuries to the triangular fibrocartilage complex (TFCC). However, the effect of ulnar shortening osteotomy on the changes in shape of the TFCC is unclear. In our study, quantitative evaluations were performed using MRI to clarify the effect of ulnar shortening on triangular fibrocartilage (TFC) thickness attributable to disc regeneration of the TFC and TFC angle attributable to the suspension effect of ulnar shortening on the TFC.

Questions/purposes The purposes of this study were (1) to compare preoperative and postoperative TFC thickness

Each author certifies that he or she, or a member of his or her immediate family, has no funding or commercial associations (eg, consultancies, stock ownership, equity interest, patent/licensing arrangements, etc) that might pose a conflict of interest in connection with the submitted article.

All ICMJE Conflict of Interest Forms for authors and Clinical Orthopaedics and Related Research editors and board members are on file with the publication and can be viewed on request.

Each author certifies that his or her institution approved the human protocol for this investigation, that all investigations were conducted in conformity with ethical principles of research, and that informed consent for participation in the study was obtained.

This work was performed at the Department of Orthopaedic Surgery, School of Medicine, Keio University, Tokyo, Japan.
\end{abstract}

Y. Yamanaka, T. Nakamura ( $₫)$, K. Sato, Y. Toyama Department of Orthopaedic Surgery, School of Medicine, Keio University, 35 Shinanomachi, Shinjuku-ku,

Tokyo 160-8582, Japan

e-mail: tosiyasu@sc.itc.keio.ac.jp; toshiyasu@ae.em-net.ne.jp

Y. Yamanaka

Department of Orthopaedic Surgery, School of Medicine, University of Occupational and Environmental Health, Fukuoka, Japan and TFC angle on MR images to quantitatively evaluate the effect of ulnar shortening osteotomy on disc regeneration and the suspension effect on the TFC; and (2) to assess whether changes in TFC thickness and TFC angle correlated with the Mayo wrist score.

Methods Between 1995 and 2008, 256 patients underwent ulnar shortening osteotomy for TFCC injuries. The minimum followup was 24 months (mean, 51 months; range, 24-210 months). A total of 79 patients $(31 \%)$ with complete followup including preoperative and postoperative MR images and the Mayo wrist score was included in this retrospective study. Evaluation of the postoperative MR images and the Mayo wrist score were performed at the final followup. The remaining 177 patients did not undergo postoperative MRI, or they had a previous fracture, large tears of the disc proper, or were lost to followup. Two orthopaedists, one of whom performed the surgeries, measured the TFC thickness and the TFC angle on coronal MR images before and after surgery for each patient. Correlations of the percent change in the TFC thickness and the magnitude of TFC angle change with age, sex, postoperative MR images, extent of ulnar shortening, preoperative ulnar variance, and postoperative Mayo wrist score were assessed.

Results Stepwise regression analysis showed a correlation between the percent change in TFC thickness and preoperative ulnar variance $\left(\mathrm{R}^{2}=0.21 ; \beta=-0.33 ; 95 \%\right.$ $\mathrm{CI},-0.11$ to $0.01 ; \mathrm{p}=0.01)$ and between the magnitude of change in the TFC angle and the extent of ulnar shortening $\left(\mathrm{R}^{2}=0.18 ; \beta=-0.29 ; 95 \% \mathrm{CI},-5.8\right.$ to $\left.0.29 ; \mathrm{p}=0.03\right)$. The Mayo wrist score was not correlated with the percent change in TFC thickness or the magnitude of change in the TFC angle.

Conclusions These results suggest that, in patients with TFCC injury with a smaller preoperative ulnar variance, 
a high residual potential for regeneration in the disc proper was seen after ulnar shortening osteotomy, and correlated with the extent of ulnar shortening and the suspension effect on TFC. However, there was no correlation between disc regeneration or the suspension effect on TFC and the Mayo wrist score.

Level of Evidence Level IV, therapeutic study. See the Instructions for Authors for a complete description of levels of evidence.

\section{Introduction}

Ulnar shortening osteotomy accompanied by decompression between the ulna and the carpal bones has been shown to help stabilize the distal radioulnar joint (DRUJ) [11]. This operation is indicated in a broad range of cases, including degenerative tears of the triangular fibrocartilage complex (TFCC), and mild to moderate DRUJ instability $[2,4,9]$. Several studies have been published concerning the use of ulnar shortening osteotomy in the treatment of TFCC injury [2, 9, 13]. These studies were based on evaluation of clinical results and arthroscopic findings.

Ulnar shortening osteotomy for TFCC injury may affect triangular fibrocartilage (TFC) thickness likely as disc regeneration of the TFC [13, 15], and the TFC angle as a suspension effect of ulnar shortening on the TFC [11]. When these structural changes on thickness and angle of the TFC may last, the effect of ulnar shortening can be retained. However, there have been no reports, to our knowledge, that the structural changes on the TFC remains after ulnar shortening.
The purposes of this study were (1) to compare on MR images preoperative and postoperative TFC thickness and TFC angle to evaluate quantitatively the effect of ulnar shortening osteotomy on disc regeneration and on the suspension effect of the TFC; and (2) to assess whether changes in TFC thickness and TFC angle correlated with the Mayo wrist score.

\section{Materials and Methods}

A retrospective followup study of preoperative and postoperative MR images of patients who had ulnar shortening osteotomy was performed. Our institutional ethics committee approved this study design.

Between 1995 and 2008, 470 patients underwent surgery for injuries to the TFCC. During that time, the general indications for ulnar shortening osteotomy were subjective symptoms of pain at the ulnar side of the wrist, objective abnormal findings of the ulnocarpal stress test, and evidence of dynamic DRUJ instability, such as a painful click. TFCC injury was diagnosed based on residual pain even after immobilization of the wrist or wearing a brace for a minimum of 3 months, which acted to reduce DRUJ instability. Contrast dye leakage from the radiocarpal joint in the DRUJ during wrist arthrography also served as a diagnostic tool for TFCC injury. Contraindications to surgery included partial resection of the TFCC in patients with TFCC injury without DRUJ instability, TFCC suture repair for patients in whom the suspension effect of the TFCC by the ulnar shortening osteotomy could not be expected because the radioulnar ligament was detached from the

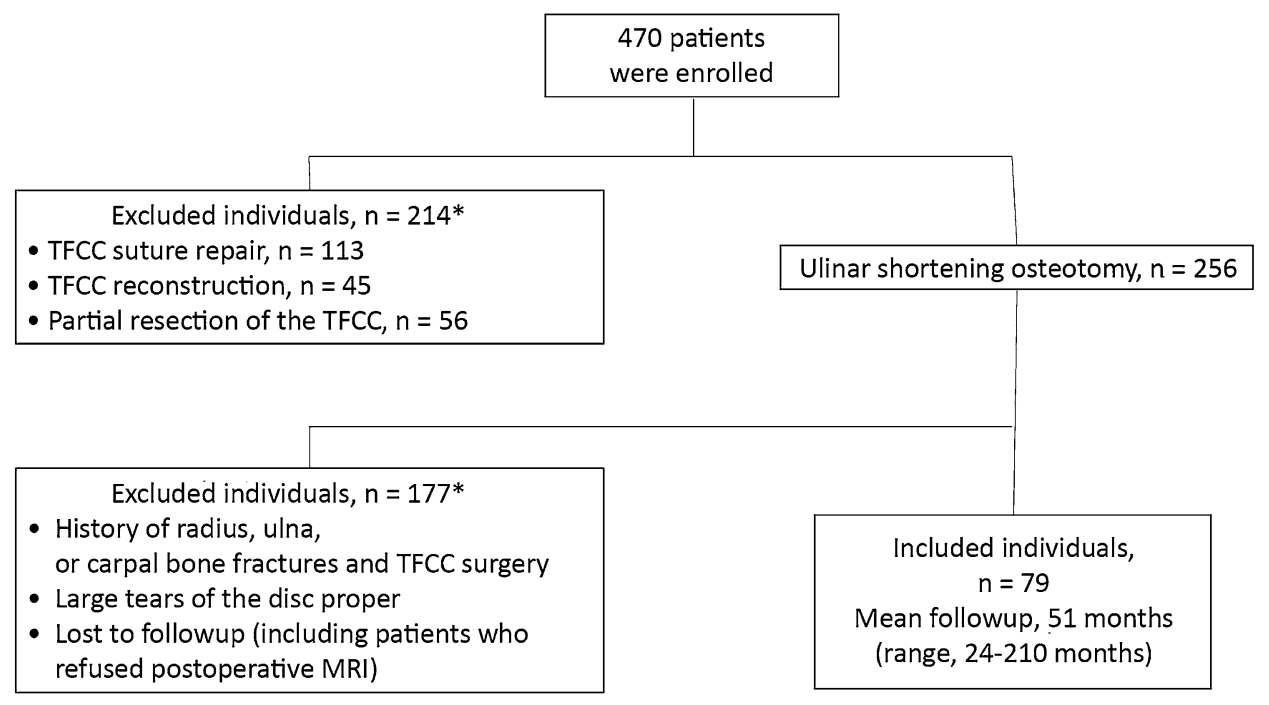

Fig. 1 The flow chart shows patient selection from 1995 to 2008 (*some individuals had two or more conditions). TFCC $=$ triangular fibrocartilage complex. 
ulnar fovea, and TFCC reconstruction for patients who could not undergo repair of the TFCC. Among patients undergoing ulnar shortening, patients with a history of radius, ulna, or carpal bone fractures and TFCC surgery,
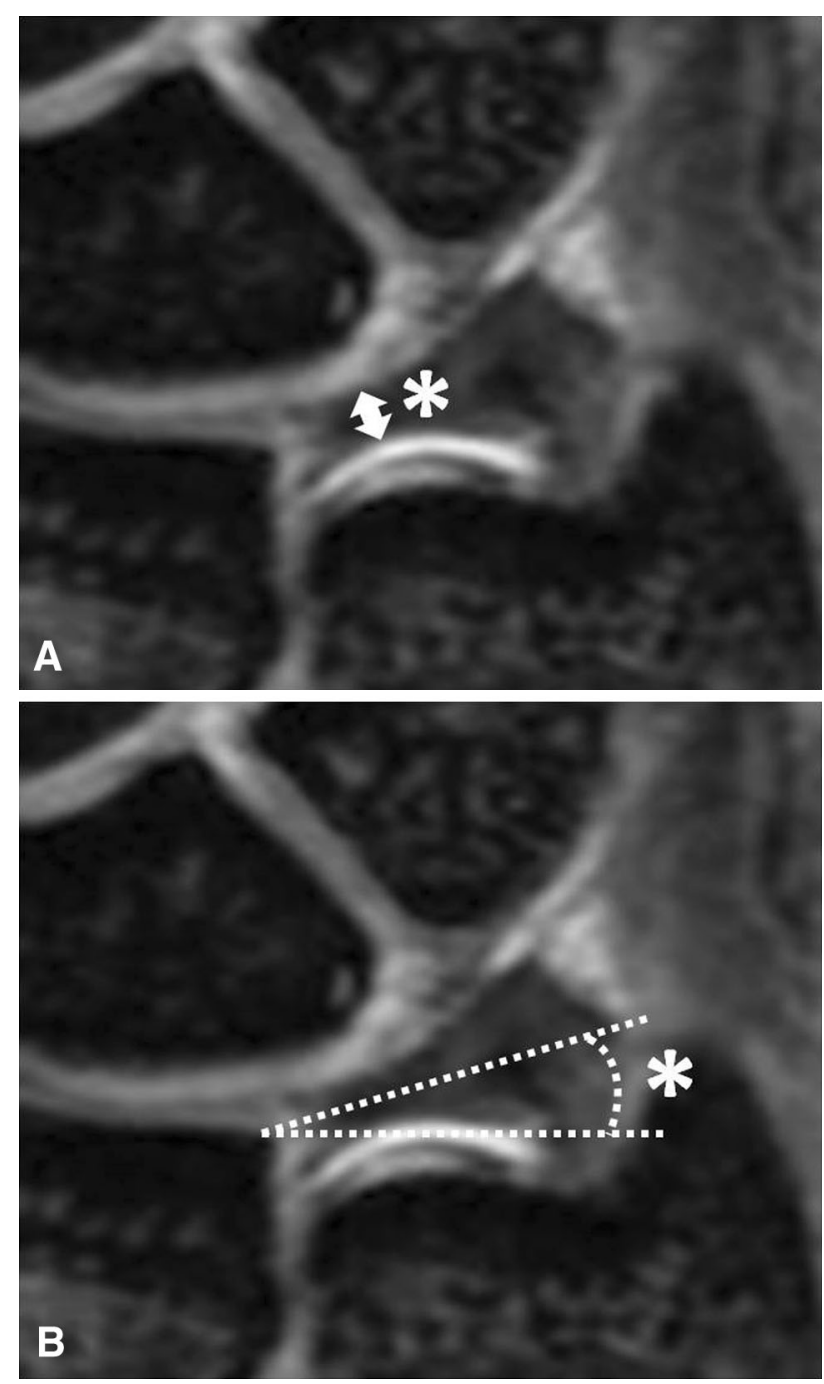

Fig. 2A-B The method for measuring TFC thickness and TFC angle on MR images is shown. (A) TFC thickness is measured at the central thinnest part of the TFC disc proper (marked by an asterisk). (B) The TFC angle is formed by a straight line drawn parallel to the horizontal line from the radius attachment point of the TFCC to the center of the thinnest part of the TFC disc (marked by an asterisk). patients who could not be evaluated by MRI because there was a large tear of the disc proper, and patients lost to followup also were excluded.

The ulnar shortening osteotomy was performed in 256 of 470 patients. As part of routine care, postoperative MR images were obtained; of the patients who underwent ulnar shortening osteotomy, $79(31 \%)$ were available for clinical examination and MRI at a median of 51 months (range, 24-210 months) postoperatively in this retrospective study (Fig. 1). These patients consisted of 39 males and 40 females (including two female patients who underwent bilateral surgery) with a mean age of 40 years (range, 15-73 years) at the time of surgery. All surgeries were performed by a senior orthopaedic hand surgeon (TN). Arthroscopic examination of the TFCC was performed through the original distal DRUJ portal, just proximal to the TFC to evaluate the joint surface of the DRUJ and the radioulnar ligament attaching to the ulnar fovea using a 1.9-mm oblique wrist arthroscope in all patients during the ulnar shortening osteotomy. In all patients with a TFCC split tear, the tear was debrided to a stable peripheral margin. For patients with degeneration and fibrillation without tears, only the fibrillation was resected arthroscopically. Ulnar shortening procedures included transverse parallel osteotomy and fixation with a 5- to 6-hole, 3.5-mm, dynamic compression plate. The extent of ulnar shortening was set at 2 to $2.5 \mathrm{~mm}$ in patients in whom the preoperative ulnar variance was neutral or negative up to $1 / 2$ the longitudinal length of the sigmoid notch to avoid over-shortening the ulna and at a level equivalent to the "neutral variance" in patients in which the preoperative ulnar variance was positive. TFCC injury classification was based on the Palmer classification [12] and was Class 1A in 20, Class 1B in two, Class 1C in two, Class 1D in 14, and Class 2 in 43 patients (including two patients with bilateral involvement, one patient had Class $1 \mathrm{~A}$ and Class 1D and the other had Class 2 for both wrists). The mean extent of ulnar shortening was $2.3 \mathrm{~mm}(2 \mathrm{~mm}$ in 61patients, $2.5 \mathrm{~mm}$ in four patients, $3 \mathrm{~mm}$ in 11 patients, $3.5 \mathrm{~mm}$ in two patients, $4 \mathrm{~mm}$ in two patients, and $5 \mathrm{~mm}$ in one patient) (including two patients with bilateral involvement, one patient had $2 \mathrm{~mm}$ and $3 \mathrm{~mm}$ and the other had $2 \mathrm{~mm}$ for both wrists). The surgeon (TN)

Table 1. Preoperative and postoperative TFC thickness and angle

\begin{tabular}{|c|c|c|c|c|c|c|c|}
\hline \multirow[t]{2}{*}{ Measurements } & \multicolumn{2}{|c|}{ TFC thickness $(\mathrm{mm})$} & \multirow{2}{*}{$\begin{array}{l}\text { Percent change in } \\
\text { TFC thickness }\end{array}$} & \multicolumn{2}{|c|}{ TFC angle (degrees) } & \multirow{2}{*}{$\begin{array}{l}\text { Magnitude of change in } \\
\text { TFC angle (degrees) }\end{array}$} & \multirow{2}{*}{$\begin{array}{l}\text { Preoperative ulnar } \\
\text { variance }(\mathrm{mm})\end{array}$} \\
\hline & Preoperative & Postoperative & & Preoperative & Postoperative & & \\
\hline Mean \pm SD & $1.3 \pm 0.4$ & $1.4 \pm 0.5$ & $0.2 \pm 0.2$ & $25 \pm 8$ & $19 \pm 7$ & $-6 \pm 6$ & $1.0 \pm 1.3$ \\
\hline Maximum & 2.1 & 2.4 & 0.6 & 58 & 43 & 4 & 5.5 \\
\hline Minimum & 0.4 & 0.5 & -0.5 & 11 & 4 & -31 & -1.1 \\
\hline
\end{tabular}

$\mathrm{TFC}=$ triangular fibrocartilage 
performed a wrist evaluation on the basis of the Mayo wrist scoring system [3] at the final followup, and postoperative MRI was performed at the same time.

MRI was performed with a $1.5-\mathrm{T}$ scanner $\left(\mathrm{Signa}^{\mathrm{TM}}\right.$ 1.5T; GE Healthcare, Milwaukee, WI, USA) with a highresolution eight-channel surface coil for the hand and wrist. The patient was positioned prone, the hand was elevated in the neutral position of the forearm and wrist in the surface coil, and the elbow was flexed at $90^{\circ}$ during imaging. For each patient, the preoperative three-dimensional T2*-weighted fat-suppressed coronal MR images (repetition time/echo time of 21/9.8 ms) with 1-mm thickness of the slice with no gap were used to check for ulnar variance and to measure the TFC thickness at the central, thinnest part of the TFC disc proper. Eleven to 13 coronal slices of the TFCC were obtained. The TFC angle formed by a straight line drawn parallel to the horizontal line from the radius attachment point of the TFCC to the center of the thinnest part of the TFC disc also was measured (Fig. 2). The TFC thickness and angle measurements were repeated on the postoperative MRI. The MR images that most clearly showed the entire TFCC including the radial and ulnar attachments were selected. In addition, the images selected for comparison preoperatively and postoperatively were those in which the TFCC, scaphoid, lunate, radius, and ulnar morphologic features matched.

The percent change in TFC thickness was calculated by dividing the difference between the preoperative and postoperative TFC thicknesses by the preoperative TFC thickness. The magnitude of the change in the TFC angle was calculated by subtracting the preoperative TFC angle from the postoperative TFC angle. Preoperative and postoperative TFC thicknesses and angle were evaluated independently by two orthopaedists (TN, YY) experienced in MRI measurements of the TFCC using ImageJ software (National Institutes of Health, Bethesda, MD, USA). One was the senior surgeon (TN), and the other was an orthopaedist with 7 years of experience (YY), and this surgeon (YY) evaluated twice with a 2-week interval. Preoperative ulnar variance also was evaluated twice with a 2-week interval by the one orthopaedist (YY).

The intraclass correlation coefficient (ICC) [7] was used to calculate the level of agreement among two or more continuous variables. An observer (YY) blinded to each patient's clinical status compared the percent change in the TFC thickness and the magnitude of change in the TFC angle with age, sex, postoperative MR images, extent of ulnar shortening, preoperative ulnar variance, and postoperative Mayo wrist score at final followup with bivariate Pearson's correlation using a simple linear regression model and stepwise regression analysis.
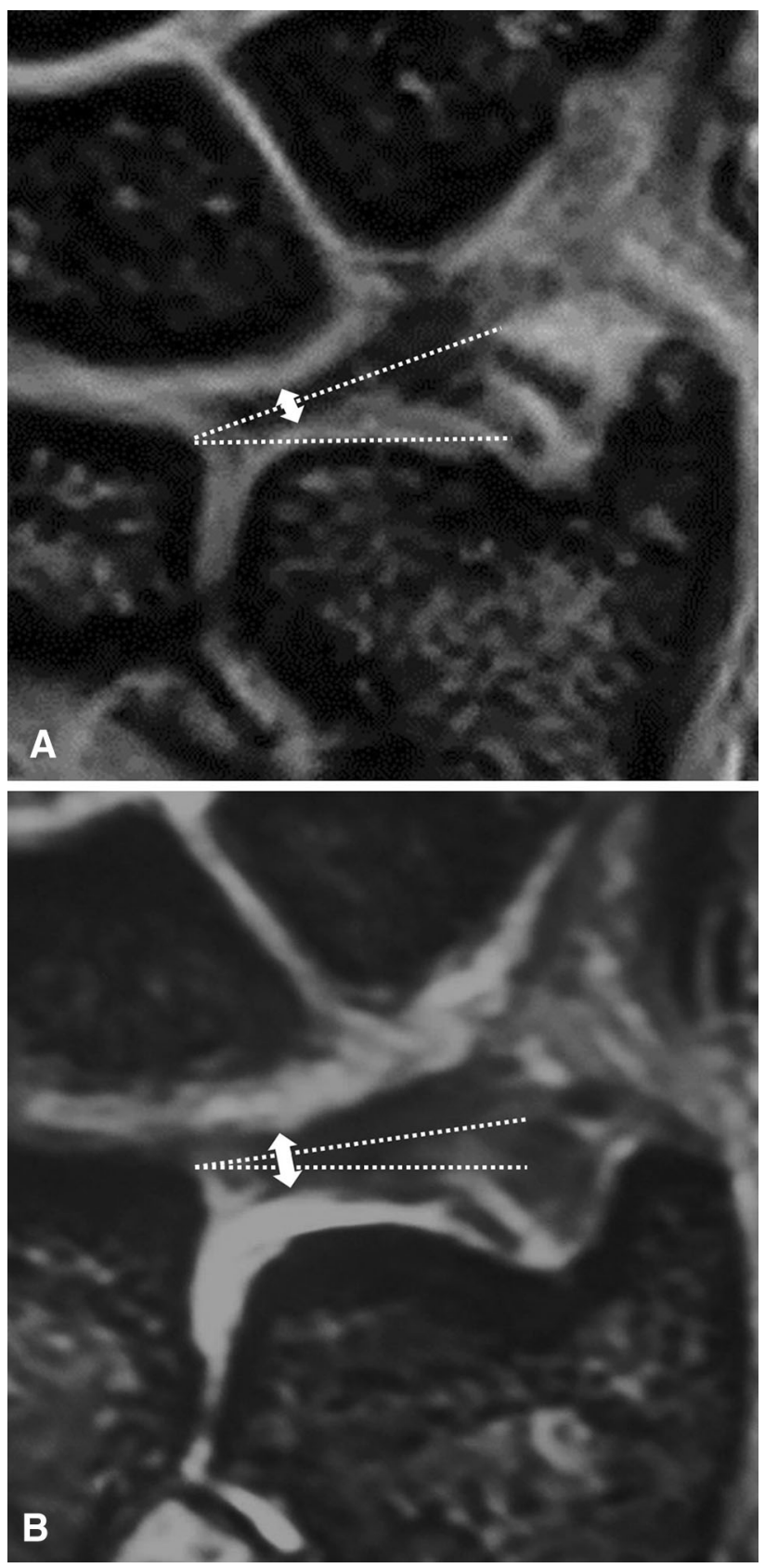

Fig. 3A-B A 42-year-old woman presented with a Palmer Class 2A TFCC injury. (A) Her preoperative, three-dimensional, T2*-weighted, fat-suppressed coronal MR image is shown. She had ulnar variance of $-0.3 \mathrm{~mm}$, TFC thickness of $1.8 \mathrm{~mm}$, and a TFC angle of $20^{\circ}(\mathbf{B})$ An MR image obtained 40 months after 2-mm ulnar shortening osteotomy is shown. The patient's TFC thickness had increased from $1.8 \mathrm{~mm}$ to $2.3 \mathrm{~mm}$, and the TFC angle decreased from $20^{\circ}$ to $8^{\circ}$.

\section{Results}

The mean intraobserver ICC was 0.94 (95\% CI, 0.62-0.98) for TFC thickness, 0.95 (95\% CI, 0.93-0.96) for TFC angle, and 0.97 (95\% CI, 0.95-0.98) for preoperative ulnar 
Table 2. Simple linear regression analysis and multiple linear stepwise regression analysis

\begin{tabular}{|c|c|c|c|c|c|c|c|c|}
\hline \multirow[t]{3}{*}{ Variable } & \multicolumn{4}{|c|}{ Simple linear regression analysis } & \multicolumn{4}{|c|}{ Multiple linear stepwise regression analysis } \\
\hline & \multicolumn{2}{|c|}{$\begin{array}{l}\text { Percent change } \\
\text { in TFC } \\
\text { thickness }\end{array}$} & \multicolumn{2}{|c|}{$\begin{array}{l}\text { Magnitude of } \\
\text { change in TFC } \\
\text { angle }\end{array}$} & \multicolumn{2}{|c|}{$\begin{array}{l}\text { Percent change in TFC thickness } \\
\left(\mathrm{R}^{2}=0.21\right)\end{array}$} & \multicolumn{2}{|c|}{$\begin{array}{l}\text { Magnitude of change in TFC angle } \\
\left(\mathrm{R}^{2}=0.18\right)\end{array}$} \\
\hline & $\mathrm{R}$ & $\mathrm{p}$ value & $\mathrm{R}$ & $\mathrm{p}$ value & $\beta(95 \% \mathrm{CI})$ & $\mathrm{p}$ value & $\beta(95 \% \mathrm{CI})$ & $\mathrm{p}$ value \\
\hline Age (years) & 0.18 & 0.10 & 0.08 & 0.49 & $-0.07(-0.005$ to 0.003$)$ & 0.51 & $0.17(-0.03$ to 0.19$)$ & 0.14 \\
\hline Sex & - & - & - & - & $-0.001(-0.11$ to 0.11$)$ & 0.99 & $0.03(-2.5$ to 3.2$)$ & 0.80 \\
\hline Postoperative MRI (months) & 0.04 & 0.75 & 0.17 & 0.14 & $-0.014(-0.002$ to 0.002$)$ & 0.90 & $0.14(-0.02$ to 0.009$)$ & 0.20 \\
\hline Extent of ulnar shortening (mm) & 0.34 & 0.002 & 0.34 & 0.002 & $-0.13(-0.16$ to 0.06$)$ & 0.31 & $-0.29(-5.8$ to 0.29$)$ & 0.03 \\
\hline Preoperative ulnar variance $(\mathrm{mm})$ & 0.43 & $<0.001$ & 0.26 & 0.02 & $-0.33(-0.11$ to 0.01$)$ & 0.01 & $-0.16(-1.96$ to 0.49$)$ & 0.23 \\
\hline Mayo Wrist Score & 0.08 & 0.47 & 0.07 & 0.55 & $0.074(-0.11$ to 0.21$)$ & 0.50 & $-0.004(-4.4$ to 4.3$)$ & 0.97 \\
\hline
\end{tabular}

$\mathrm{TFC}=$ triangular fibrocartilage $; \mathrm{R}=$ correlation coefficient; $\beta=$ standardized correlation coefficients $(95 \% \mathrm{CI}) ; \mathrm{R}^{2}=$ adjusted determination coefficient.

variance. The mean interobserver ICC was $0.87(95 \% \mathrm{CI}$, 0.82-0.91) for TFC thickness and 0.93 (95\% CI, 0.90-0.95) for TFC angle.

The mean preoperative TFC thickness and TFC angle measurements were $1.3 \mathrm{~mm}$ and $25^{\circ}$, respectively. Alternatively, the mean postoperative TFC thickness and TFC angles were $1.4 \mathrm{~mm}$ and $19^{\circ}$, respectively. The mean percent change in TFC thickness and magnitude of change in the TFC angle were $0.2^{\circ}$ and $-6^{\circ}$, respectively. The mean preoperative ulnar variance was $1.0 \mathrm{~mm}$ (Table 1).

Stepwise regression analysis showed correlations between the percent change in the TFC thickness and preoperative ulnar variance $\left(\mathrm{R}^{2}=0.21 ; \beta=-0.33 ; 95 \% \mathrm{CI}\right.$, -0.11 to $0.01 ; \mathrm{p}=0.01$ ) and between the magnitude of change in the TFC angle and the extent of ulnar shortening $\left(\mathrm{R}^{2}=0.18 ; \beta=-0.29 ; 95 \% \mathrm{CI},-5.8\right.$ to $\left.0.29 ; \mathrm{p}=0.03\right)$ (Fig. 3). Seventy patients had excellent results and nine had good results based on the Mayo wrist score. The Mayo Wrist Score did not correlate with the percent change in TFC thickness or magnitude of the change in the TFC angle (Table 2). One patient had nonunion at the ulnar shortening osteotomy site; refixation was performed 6 months after initial surgery and bone union was achieved.

\section{Discussion}

Several studies have been published concerning the use of ulnar shortening osteotomy for treatment of TFCC injury $[2,9,13]$. However, these studies were based on evaluation of clinical results and arthroscopic findings. Therefore, in our study, quantitative evaluations were performed using MRI to clarify the effect of ulnar shortening on TFC thickness attributable to disc regeneration of the TFC and the TFC angle attributable to the suspension effect of ulnar shortening on the TFC. In our study, patients with a large ulnar variance before surgery were found to be unlikely to show TFC regeneration after surgery. Furthermore, patients with more ulnar shortening were more likely to show a suspension effect.

There are limitations to our study. First, we evaluated only 79 of 256 patients who underwent ulnar shortening osteotomy; therefore, it is possible that the results of this study are not applicable to all patients with a TFCC injury. However, since 81 cases (two of the 79 patients had bilateral surgery) were evaluated, the number was sufficient for quantitative evaluation. Second, in the measurements of TFC thickness and angle, interobserver reliability was evaluated for two raters (TN, YY), whereas intraobserver reliability was evaluated for only one rater (YY). However, interobserver reliability is more clinically useful than intraobserver reliability, and if researchers document an acceptable level of interobserver reliability in the appropriate context, no further reliability testing is necessary [5]. Third, the surgeon (TN) evaluated preoperative and postoperative TFC thicknesses, the TFC angles, and the Mayo Wrist Score at final followup. However, assessor bias was unlikely because strict criteria were used to measure MR images.

The avascular central zone of the TFCC was reported to have no healing potential [1]. However, some have reported that the degenerative disc has the potential for regeneration, especially in patients with neutral or negative ulnar variance, suggesting that an ulnar shortening osteotomy may stimulate disc regeneration $[13,15]$. In one study, postoperative rearthroscopy showed healing of the injured central portion of the TFCC in 50\% of patients who underwent ulnar shortening osteotomy for a TFCC injury [13]. Postoperative rearthroscopy also revealed TFCC tear healing in 10 of 13 patients who underwent an ulnar shortening osteotomy for ulnocarpal abutment syndrome [14]. The number of apoptotic cells in the degenerative disc increases with increasing values of ulnar variance. Preventing apoptosis of the disc cells may be possible if the variance can be reduced by an ulnar shortening osteotomy [16]. 
Our study showed that the percent change in the TFC thickness after surgery was correlated with the preoperative ulnar variance. The percent change in the TFC thickness was greater than 0 in $59(88 \%)$ of the 67 patients with preoperative ulnar variance less than $2 \mathrm{~mm}$, suggesting that regeneration of the disc proper had likely occurred in these patients. However, the percent change in TFC thickness was 0 or less in $10(71 \%)$ of the 14 patients with preoperative ulnar variance of $2 \mathrm{~mm}$ or more, suggesting that regeneration of the disc proper after ulnar shortening is unlikely to have occurred in these patients. Apoptosis of the cells in the disc proper might already have taken place before the ulnar shortening osteotomy in patients with positive values for preoperative ulnar variance [16], so that regeneration after the operation was unlikely to occur in these patients. In our patients with TFCC injury and ulnar variance values less than $2 \mathrm{~mm}$, a high residual potential for regeneration in the disc proper was seen after ulnar shortening osteotomy with the possibility of increasing postoperative disc thickness. In our patients, the TFC angle decreased after ulnar shortening osteotomy. A decrease of the TFC angle may be the result of the continuous suspension effect on the TFCC after ulnar shortening osteotomy. A negative correlation was seen between the extent of ulnar shortening and the magnitude of change in the TFC angle. This confirmed the pulling down of the TFCC along the major axis resulting from ulnar shortening. Nishiwaki et al. [11] reported that the suspension effect on the TFCC increases as the extent of ulnar shortening increases, resulting in greater stabilization of the DRUJ, as long as the radioulnar ligament remains attached to the ulnar fovea. However, the outcome did not always improve as the extent of ulnar shortening increased [11]. Loh et al. [8] reported that ulnar shortening beyond the neutral level in patients with positive ulnar variance, or shortening beyond $2.5 \mathrm{~mm}$ in patients with negative ulnar variance, was not favorable in view of the morphologic features of the DRUJ. Nishiwaki et al. [10] also stated that it would be desirable to keep the extent of ulnar shortening to within $3 \mathrm{~mm}$ to prevent arthritic changes in the DRUJ arising from the elevated pressure.

Some authors reported good clinical results after ulnar shortening osteotomy for TFCC tears with a positive ulnar variance $[6,14,17]$. We found no relationships between the Mayo Wrist Score and TFC thickness or the TFC angle on MR images; however, all our patients had excellent or good outcomes based on the Mayo Wrist Score. Our findings were similar to those of previous studies $[6,14$, 17], indicating that ulnar shortening osteotomy is an effective treatment for TFCC tears.

The results of our study suggest that, in patients with TFCC injury and ulnar variance less than $2 \mathrm{~mm}$, there is a high residual potential for regeneration in the disc proper after ulnar shortening osteotomy, with the possibility of increasing postoperative disc thickness, and the suspension effect on the TFCC correlated with the extent of ulnar shortening. However, there was no relationship between disc regeneration or the suspension effect on the TFCC and the Mayo Wrist Score.

\section{References}

1. Bednar JM. Arthroscopic treatment of triangular fibrocartilage tears. Hand Clin. 1999;15:479-488, ix.

2. Boulas HJ, Milek MA. Ulnar shortening for tears of the triangular fibrocartilaginous complex. J Hand Surg Am. 1990;15:415-420.

3. Cooney WP, Linscheid RL, Dobyns JH. Triangular fibrocartilage tears. J Hand Surg Am. 1994;19:143-154.

4. Darrow JC Jr, Linscheid RL, Dobyns JH, Mann JM 3rd, Wood MB, Beckenbaugh RD. Distal ulnar recession for disorders of the distal radioulnar joint. J Hand Surg Am. 1985;10:482-491.

5. Karanicolas PJ, Bhandari M, Kreder H, Moroni A, Richardson M, Walter SD, Norman GR, Guyatt GH. Collaboration for Outcome Assessment in Surgical Trials (COAST) Musculoskeletal Group. Evaluating agreement: conducting a reliability study. J Bone Joint Surg Am. 2009;91(suppl 3):99-106.

6. Kim BS, Song HS. A comparison of ulnar shortening osteotomy alone versus combined arthroscopic triangular fibrocartilage complex debridement and ulnar shortening osteotomy for ulnar impaction syndrome. Clin Orthop Surg. 2011;3:184-190.

7. Landis JR, Koch GG. The measurement of observer agreement for categorical data. Biometrics. 1977;33:159-174.

8. Loh YC, Van Den Abbeele K, Stanley JK, Trail IA. The results of ulnar shortening for ulnar impaction syndrome. J Hand Surg Br. 1999;24:316-320.

9. Minami A, Kato H. Ulnar shortening for triangular fibrocartilage complex tears associated with ulnar positive variance. $J$ Hand Surg Am. 1998;23:904-908.

10. Nishiwaki M, Nakamura T, Nagura T, Toyama Y, Ikegami H. Ulnar-shortening effect on distal radioulnar joint pressure: a biomechanical study. J Hand Surg Am. 2008;33:198-205.

11. Nishiwaki M, Nakamura T, Nakao Y, Nagura T, Toyama $Y$. Ulnar shortening effect on distal radioulnar joint stability: a biomechanical study. J Hand Surg Am. 2005;30:719-726.

12. Palmer AK. Triangular fibrocartilage complex lesions: a classification. J Hand Surg Am. 1989;14:594-606.

13. Tatebe M, Horii E, Nakao E, Shinohara T, Imaeda T, Nakamura $\mathrm{R}$, Hirata $\mathrm{H}$. Repair of the triangular fibrocartilage complex after ulnar-shortening osteotomy: second-look arthroscopy. J Hand Surg Am. 2007;32:445-449.

14. Tatebe M, Shinohara T, Okui N, Yamamoto M, Hirata H, Imaeda T. Clinical, radiographic, and arthroscopic outcomes after ulnar shortening osteotomy: a long-term follow-up study. J Hand Surg Am. 2012;37:2468-2474.

15. Unglaub F, Thomas SB, Wolf MB, Dragu A, Kroeber MW, Mittlmeier T, Horch RE. Cartilage cell proliferation in degenerative TFCC wrist lesions. Arch Orthop Trauma Surg. 2010; 130:953-956.

16. Unglaub F, Wolf MB, Thome MA, Germann G, Sauerbier M, Reiter A. Correlation of ulnar length and apoptotic cell death in degenerative lesions of the triangular fibrocartilage. Arthroscopy. 2008;24:299-304.

17. Wolf MB, Kroeber MW, Reiter A, Thomas SB, Hahn P, Horch RE, Unglaub F. Ulnar shortening after TFCC suture repair of Palmer type 1B lesions. Arch Orthop Trauma Surg. 2010; 130:301-306. 